\title{
Monitoring Immune Cell Infiltration in the Brain with Intravital Two-Photon Microscopy
}

\author{
D.S. Barkauskas**, Y. Othman*, A.Y. Huang*.
}

* Department of Pediatrics, Case Western Reserve University, 10900 Euclid Ave, Cleveland, OH, 44106; ** Department of Biomedical Engineering, Case Western Reserve University, 10900 Euclid Ave, Cleveland, $\mathrm{OH}, 44106$

Although widely used in laboratory research, experimental autoimmune encephalitis (EAE) is a mouse model of multiple sclerosis many aspects of which have not been fully described. Limitations in current techniques to capture rare immune cell events in space and time have hindered the characterization of the initiation and progression in EAE. Advances have been made in our ability to visualize these processes on the cellular level with the advent of two-photon microscopy. The cranial window, described by Mostany [1], has allowed serial imaging of the brain over time with minimal disruption to the tissue environment.

The interplay between three cell types in EAE has not yet been elucidated: 1) microglia, brain resident immune surveillance cells; 2) astrocytes, which are critical to the maintenance of the blood brain barrier (BBB); and, 3) peripherally activated immune cells, particularly myelin-specific, $\mathrm{CD} 4^{+}$ IL17-producing Th17 cells. We hypothesize that the initial cellular interaction responsible for Th17 cell infiltrate through the intact blood-brain barrier during EAE induction to be similar to salmonella infection, where it has been shown that dendritic cells are able to sample the gut lumen to interact directly with the pathogen [2]. We attempt to show that brain resident immune cells, microglia and perivascular antigen presenting cells are responsible for the infiltration of myelin specific $\mathrm{T}$ cells into the brain parenchyma by sampling the immune cells in the blood stream across the blood brain barrier.

In these experiments we use CD11c-GFP and ubiquitin-CFP myelin oligodendrocyte glycoprotein (MOG) specific transgenic mice. A craniotomy was sealed with a \#1.5 cover glass bonded to the skull with vetbond. Dental acrylic was used to create a permanent well to maintain the fluid needed with an immersion objective. The mouse received dexamethasone and carprofen for pain relief during and immediately after surgery. 4 days after the surgery, $3 \times 10^{6}$ myelin specific $\mathrm{T}$ cells were injected intravenously into the mouse. 24 hours later, EAE was induced via peripheral immunization with MOG-peptide admixed in CFA, and the progression of disease was imaged for 1 hour each day over the first 12 days of disease. A $150 \mathrm{kDa}$ fluorescently labeled dextran was used to visualize blood vessels and $\mathrm{BBB}$ permeability.

Two-photon microscopy demonstrated transient leaks in BBB and the accumulation of immune cells through the progression of disease (Figure 1). We also postulate that the migration pattern of infiltrating $\mathrm{T}$ cells correlates well with the focal lesion characteristics of multiple sclerosis (Figure 2). The long term imaging of this disease progression was made possible with the cranial window, two-photon microscopy and fluorescent reporter mice [3]. Future work includes further defining cellular components in the peri-vascular microenvironment of the BBB which are responsible for attracting MOG-specific Th17 cells to cross the vascular barrier into the CNS. 


\section{References}

[1] R. Mostany, C. Portera-Cailliau, J Visualized Exper. 12, http://www.jove.com/index/Details.stp?ID=680 (2008).

[2] M. Chieppa et. al., J Exp Med. 203:13 (2006) 2841.

[3] This research was supported by the Dana Foundation "Brain \& Immuno-Imaging" Program, the St. Baldrick's Foundation, Hyundai Motors of America "Hope-on-Wheels" Research Program, Cancer Research Institute, and the Gabrielle's Angel Foundation, Rainbow Fellow Research Award Program.
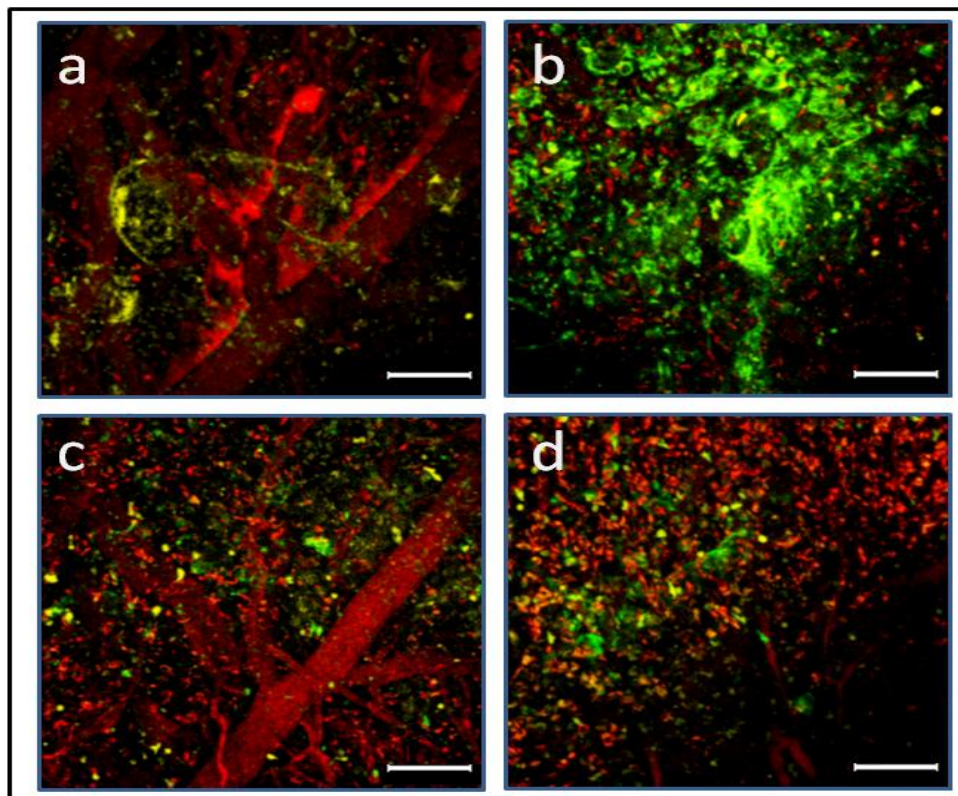

Figure 1: CD11c-GFP (green) cells in the brain, MOG specific T cells (yellow) and fluorescent dextran vessel dye (red) during EAE induction. (a) Day 3; (b) Day 6; (c) Day 9; (d) Day 12. Scale bar is 100 um.
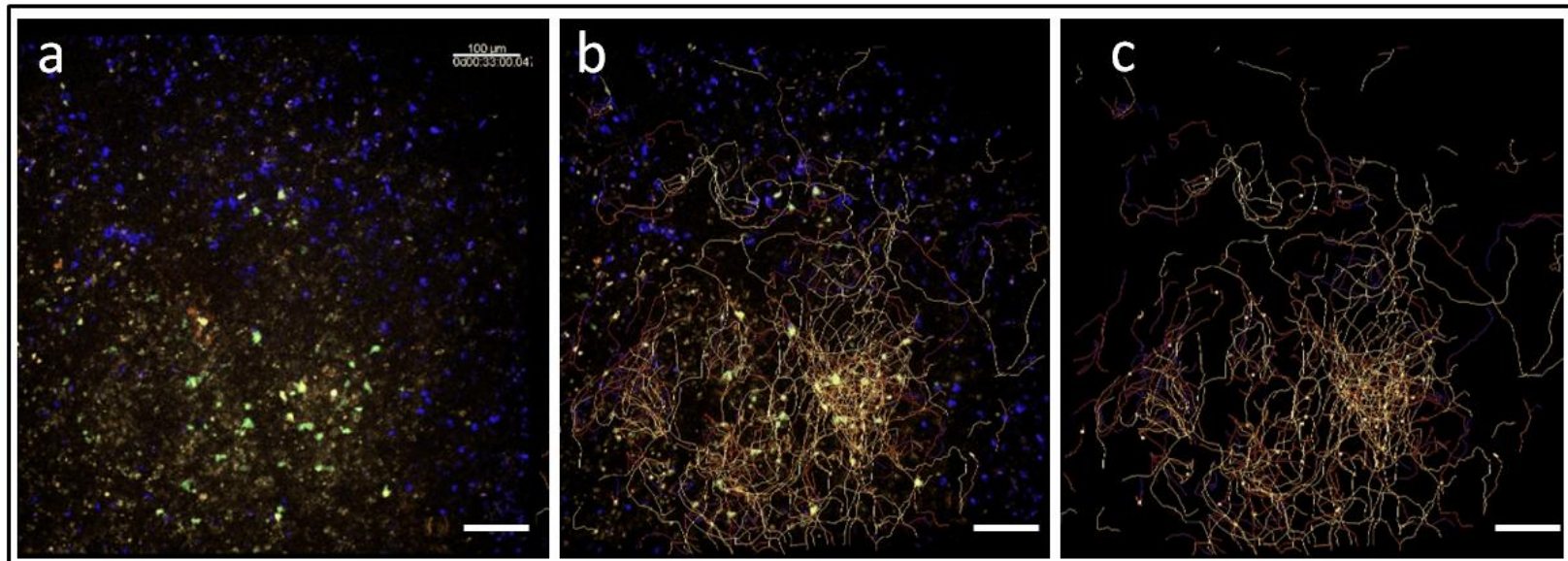

Figure 2: CD11c-GFP (orange) cells in the brain, MOG specific T cells (green) and phagocytic cells that have taken up fluorescent dextran (blue) on Day 9 of EAE induction.

(a) Concentration of T cells; (b) Merged image of cells and track information;

(c) Cell migration tracks, visualized by using Imaris Bitplane software.

Scale bar is 100 um. 